Hernández-Morales, M.M. \& Hernández-Pina, F. (2014). Los valores en la obra de Félix Varela. Revista Electrónica Interuniversitaria de Formación del Profesorado, 17 (3), 173-185.

DOI: http://dx.doi.org/10.6018/reifop. 17.3.204131

\title{
Los valores en la obra de Félix Varela
}

\author{
María Magdalena Hernández Morales ${ }^{(1)}$, Fuensanta Hernández Pina ${ }^{(2)}$ \\ ${ }^{(1)}$ Universidad de Ciego de Ávila, Cuba, ${ }^{(2)}$ Universidad de Murcia, España
}

\section{Resumen}

El trabajo ofrece un estudio del postulado ético del filósofo y pedagogo cubano de la segunda mitad del siglo XIX Félix Varela y Morales; que sirve de fundamento teórico para la formación de valores del estudiantado universitario de la carrera de Estudios Socioculturales, desde el contenido curricular en las disciplinas Teoría Filosófica y Sociopolítica e Historia, y Cultura Cubana. Tiene como objetivo identificar el modelo axiológico implícito en una de sus más importantes obras Cartas a Elpidio, a través, del estudio de su prédica justipreciando así, su vigencia. Se realiza el análisis de contenido axiológico, utilizándose el programa NUDIST en el análisis cualitativo de datos, a una muestra de cuatro epístolas del primer volumen titulado Impiedad. Se constata que en la obra esta presente un modelo axiológico educativo que puede ser utilizado en la formación de valores del profesional universitario en el contexto actual.

\section{Palabras clave}

Valores; axiología; análisis cualitativo.

\section{Human values in Félix Varela's letters}

\section{Contacto}

María Magdalena Hernández Morales, email: magdalena@rect.unica.cu, Carretera a Morón km 9, C. P. 65200, Ciego de Ávila, Cuba.

El artículo se vincula a un proyecto de investigación, devenido en tesis doctoral. 


\section{Abstract}

The paper presents a study of the ethical postulate of Felix Varela. A Cuban pedagogue from the second half of the IXX century. This study serves as a theoretical basis in the formation of values of the students majoring Sociocultural Studies throughout their curriculum contents in the disciplines Philosophical and Sociopolitical Theory and History, and Cuban Culture, during the teaching learning process. It is aimed at identifying an implicit axiological model in one of his most important works "Letters to Elpidio", analyzing the universality of his preaching to appraise this way its validity. NUDIST program is used in the qualitative data analysis applied to a sample of four epistles from the first volume entitled "Impiety". It is corroborated the presence of an axiological educational model in his work and that it can be used in the current context university professionals.

\section{Key words}

Values; axiological; qualitative analysis.

\section{Introducción}

"La educación en valores es una necesidad hoy", así lo afirma Cámara $(2010,1)$, criterio que se comparte por la importancia que adquiere no sólo para la comprensión teórica, sino para la actuación práctica.

Como parte de la cultura con un carácter esencialmente educativo, los valores representan una relación de significación entre los distintos procesos culturales y las necesidades e intereses de la sociedad en su conjunto.

En el ámbito de la actividad pedagógica, corresponde especialmente al profesorado detenerse en éstos y preguntarse cómo puede contribuir a la educación y la formación de sus educandos a partir de los contenidos de la ciencia que imparte. En esta dimensión, la formación y la educación en valores incide en las más diversas facetas de la vida.

Variados son los criterios aportados por estudiosos del tema, así Alfonso (2009) se refiere a que los valores son significaciones positivas que se adquieren partir de la práctica concreta, activa y diaria de los individuos, grupos sociales, clases y la sociedad en su conjunto.

Por su parte, Vila (2005) hace alusión a que los valores, sin olvidar su origen socio-histórico impregnados a través de la cultura.

Asimismo, Ortega y Mínguez (2001), en su estudio concluyeron que, cuando se habla de "valores en educación, (...) no cabe un discurso sin que necesariamente, de un modo explícito o implícito, estén presentes los valores. De otro modo, no habría un acto educativo" (p.14).

Igualmente Peiró (2012) señala: "educar en valores es un medio para lograr la madurez de los alumnos" (p. 1).

Evidentemente la relación entre los componentes instructivo y educativo, lleva implícito los valores, si se quiere lograr que los educandos sean agentes activos y transformadores de la realidad en que vive, este proceso deberá estar encaminado a inculcar en ellos, lo más valioso del legado histórico cultural, a fin de alcanzar las realizaciones prácticas del contexto socio histórico presente. 
De este modo, según lo expresado por Horruitiner (2006), la Universidad cubana centra su atención principal en la formación de los valores que deben caracterizar al profesional, creando espacios para alcanzar los objetivos instructivos y educativos que propicien la formación integral del estudiante.

Estas valoraciones se basan en todo el acervo histórico-cultural acumulado por las generaciones precedentes y las potencialidades que ofrece el sistema educacional para el logro de los objetivos educativos que se propone. La formación de profesionales como proceso sustantivo integral no puede ser de calidad, si no se aprecia la significación de la cultura, la apropiación de los valores heredados a través de la historia, presentes en el pensamiento y las tradiciones, con la finalidad de perfeccionar las relaciones humanas en la misma.

En la literatura consultada se puedo constatar las posibilidades que ofrecen los estudios axiológicos realizados a escritos de personalidades históricas, y su poca utilización para contribuir a la formación valores de los futuros profesionales; dentro de ellas, Félix Varela, cuyo pensamiento sienta las bases de la cultura cubana.

Sus reflexiones sirven de fuente para el estudio en las disciplinas Teoría Filosófica y Sociopolítica e Historia, y Cultura Cubana, de la especialidad de Estudios Socioculturales. Estos estudios se desarrollan en el tercer curso, subrayando el papel desempeñado por las figuras relevantes, atendiendo a diferentes aspectos de su vida y obra.

Los contenidos de las materias deben facilitar, junto con la aprehensión de los aspectos meramente instructivos, los de orden educativos, para procurar una formación integral, en el que se destacan los valores siguientes: intelectuales, el análisis, la reflexión; político: el patriotismo; moral: la responsabilidad, la justicia, la honradez, el humanismo y la dignidad; social: la solidaridad, la tolerancia.

Este es el fundamento que sirve para argumentar por qué se toma el análisis del modelo axiológico presente en el pensamiento ético del pedagogo Félix Varela, con el propósito de que las nuevas generaciones se apropien de lo mejor de su legado en la cultura cubana y lo conviertan en convicciones que pauten su actuación.

\section{¿Quién fue Félix Varela?}

Figura trascendental del siglo XIX cubano, nacido en La Habana el 20 de Noviembre de 1778. Sus padres fueron Don Francisco Varela Pérez, español y Doña Josefa Morales y Medina, cubana. A muy temprana edad queda huérfano al cuidado de su abuelo paterno, quien lo guía en su proceso de formación pretendiendo que siguiera la tradición familiar de vida militar; pero no logra tal propósito. Años más tarde Varela pide entrar a un seminario para hacerse sacerdote. Viaja en compañía de su abuelo Don Bartolomé a San Agustín de la Florida, Estados Unidos de América, e inicia sus estudios primarios con el Padre O'Reilly, quien le enseña latín, gramática y música. De la familia heredaría el amor a Cuba, el aprecio a la cultura hispana y la valentía en la defensa de sus ideas, la sensibilidad, y la ternura; de su maestro, la unión entre lo católico y lo patriótico, el gusto musical por el violín y la inquietud por el conocimiento. Muere en esta propia ciudad víctima del confinamiento al exilio, el 25 de febrero de 1853.

Realiza sus estudios para sacerdote en el Seminario San Carlos de La Habana, y estudia a la vez, en la Universidad de La Habana, siendo tan decidido y audaz en su empeño que a los 19 años comienza a heredar las cátedras de sus propios profesores.

Renovó la enseñanza de la época utilizando el español en sus clases y libros, en los que abandonó el escolasticismo imperante por la filosofía ecléctica e introdujo la 
experimentación en el estudio de las ciencias naturales. Da mucha importancia a que sus alumnos aprendan a razonar, que aprendan a pensar y a decidir por sí mismos. Por eso, el destacado maestro José de la Luz y Caballero, su discípulo, dijo: "Mientras se piense en Cuba, se pensará con respeto y veneración en el primero que nos enseñó en pensar" (1981, p.32).

La obra de Félix Varela y Morales contienen el pensamiento de un hombre que trascendió, con sus ideas y su acción, el estrecho marco de su época histórica. Un hombre cuyo ejemplo y legado debe ser estudiado en su real dimensión. Prueba de que quien fue capaz de cumplir cabalmente con los deberes correspondientes a su época, es un hombre de todas las épocas.

Desde la perspectiva educativa, la mejor manera de incidir en el proceso de formación de valores, es profundizando en el estudio de las raíces históricas y culturales que nos legara, además de sus aportes, el valor educativo de su ejemplo.

\section{La obra de Félix Varela "Cartas a Elpidio"}

Del universo que forma la obra del pedagogo, se selecciona una que por su importancia, merece un estudio profundo por la manera en que está escrita, la forma coloquial que adopta; a quién va dirigida, a la juventud; y por el mensaje intencional educativo que expresa. Se selecciona, por el valor extraordinario que posee desde diferentes aristas: filosófica, literaria, patriótica, ética, de profundo contenido educativo; es considerada como su testamento intelectual.

Es propósito, identificar a través del análisis de contenido, el modelo axiológico presente en la obra de Félix Varela s, específicamente Cartas a Elpidio, sobre la impiedad, la superstición y el fanatismo en sus relaciones con la sociedad; como una de sus mejores escritos dirigidos a la juventud en las que lega su mensaje educativo.

Como bien se señala por la investigadora Perla Cartaya Cota (1998): "El Maestro dio a sus Cartas un contenido filosófico-moral sin que faltara el componente pedagógico-psicológico, propio de su magisterio" (p. 142).

Su publicación se efectuó en 1835, año en que vio la luz el primer volumen, que trata sobre la Impiedad. El segundo libro, dedicado a la Superstición, se publicó en 1838; el tercero, que debía referirse al Fanatismo, no llegó a ver la luz, pues la indiferencia primero, y la mala voluntad después, con que fueron recibidos los dos tomos iníciales de la obra, disuadieron a Varela de entregarlo a la imprenta.

En el prólogo que acompaña al primer volumen, Varela advierte que las Cartas a Elpidio: "no contienen una defensa a la religión, aunque, por incidencia, se aprueban en ellas algunos de sus dogmas. (...) como mi objeto es advertir, quedarán inéditos el segundo y el tercer tomo, si (...) no tiene buena acogida el primero" (1996, p.2).

Incluye una amplia plataforma axiológica, de profundo contenido humanista, porque Elpidio simboliza un cuerpo moral, la población cubana, y en particular su juventud. Sea una persona o un recurso literario, como bien se discute, lo esencial es que le escribe a la juventud, a la que califica como la esperanza de la patria.

No fueron, por supuesto, el corto alcance de esta obra, la fallida selección de su problemática, o la insipidez de su autor para contarla, como ha sido señalado por Cartaya (1998), las causas de la poca suerte que tuvo su edición, fue un trabajo, que acusa la madurez del pensamiento; de extraordinaria lucidez y originalidad literarias en el abordaje de una temática que justamente se ubicaba en el centro de los conflictos socio clasistas y nacionales de la época. 
Pero esta valiosa semilla encontró que en su lugar de destino no imperaban las condiciones para germinar, pues, para la época no existía una corriente ideológica que la abrazara, a pesar de los esfuerzos de algunos patriotas y amigos del presbítero. Tales condiciones, son valoradas de manera sintética, por Eduardo Torres-Cuevas (2002), cuando señala: “(...) es una obra política de proyecciones ideológicas y sociales. (...). La obra sigue teniendo como objetivo fundamental el desarrollo moral y patriótico de la juventud cubana" (p.363).

Se trata de una idea que se comparte y se toma como referente en la formación de valores de las nuevas generaciones de cubanos junto al pensamiento martiano.

Según Gallegos (1997), “la obra Cartas a Elpidio, se adentra más en el análisis teórico, orientándose, hacia la esfera de los valores. El pensamiento vareliano se sitúa en un plano de elaboración conceptual y axiológica que no encontrará paralelo hasta la aparición del pensamiento martiano" (p.61).

Es de esperar que este salto no se produciría, si no se tiene en cuenta la eticidad legada, también, por José de la Luz y Caballero, Rafael María de Mendive y otros pensadores de la época, quienes sirvieron de nexo histórico entre Félix Varela y José Martí.

Lolo (2013), se refiere al nexo existente entre Varela y Martí advirtiendo la continuidad histórica del pensamiento legado por Varela a su discípulo Mendive, señala: "Hay, en efecto, elementos vareliano que llegaron a Martí mucho antes de éste ser capaz de comprender en toda su integridad las Cartas a Elpidio, (...) lo ayudó a desarrollarla un maestro que nunca olvidaría, Mendive (p.15).

La presencia de los valores morales de Félix Varela en su obra, se expresa tácitamente en la subordinación del bien individual al bien común, manifiesto no sólo en cada uno de sus escritos, sino en su actuar como patriota y sacerdote.

Deseaba la plena igualdad entre los hombres en cuanto a derechos y deberes sociales, para lograr la verdadera condición humana. Así escribe: “(...) aprenden los hombres a ser iguales sin dejar de ser diferentes, los ricos y los pobres, los sabios y los ignorantes, (...) todos forman una familia, todos se consideran sujetos a leyes y libres de opresión y de injusticia" (1996, p.63).

De Céspedes (1999), considera que en el dominio de la Filosofía, Varela fue renovador, ecléctico o electivo y utilitarista, capaz de incluir, en primer lugar, el cultivo de la virtud como la cualidad más útil para la persona que vive y se desarrolla en determinado marco social que, con amor, ayuda al prójimo como ente desarrollador de esa virtud.

Conviene destacar las valoraciones de Pupo (2008), cuando se refiere a la valía del pensamiento vareliano en la intelectualidad cubana, "apegado creadoramente aún a las normas de su tiempo, en cuanto a escritura, influencias, orden lógico y espíritu persuasivo de sus explicaciones" (p.2).

En cada una de sus escritos se destaca el valor de su condición humana. La obra Cartas a Elpidio es un canto a la esperanza, y traza una vía hacia la igualdad plena entre los seres humanos.

Corresponde a continuación el análisis de cada una de las epístolas escogidas a fin de significar la valía de estos escritos en la formación de valores del estudiantado universitario. 


\section{Metodología}

Con la finalidad de realizar un estudio de tipo exploratorio, dado que "el objetivo es examinar un tema o problema de investigación poco estudiado o que no ha sido abordado antes", como lo definen Hernández, Fernández y Batista, (2006, p.100), se toma como fundamento, ya que el tema en cuestión no ha sido abordado desde la perspectiva axiológica y su valía en la labor educativa del profesorado.

Se seleccionan las epístolas contenidas en la obra objeto de estudio, y se realiza el análisis de contenido axiológico a cuatro de ellas, a modo de ilustración, con la finalidad de esclarecer el modelo axiológico que en estas se manifiestan.

La metodología que describimos contiene varias fases, todas precedidas de la revisión bibliográfica. Primeramente, fue necesario realizar una lectura profunda de las obras del autor para determinar si era pertinente seleccionar las Cartas a Elpidio, como ejemplo de lo aportado por Varela a la ética, y que sirve de fundamento a las reflexiones que acompañan su selección. Por su complejidad en el análisis, se hizo necesario elaborar un glosario de términos y de aforismos para facilitar su mejor comprensión.

Todo esto permite establecer también, los criterios con los que se analiza la selección de la muestra que formaría parte del análisis que presupone la investigación. Se procede, asimismo, a la adaptación del sistema de categorías del que se parte, el Modelo Axiológico de Educación Integral, de Gervilla (2000), a la naturaleza específica del objeto de estudio, y los valores contenidos en el plan de estudio de la carrera, para el proceso de educación ética del estudiantado. Se toma como fundamento el modelo de Gervilla pues ha sido contextualizado y validado en otros estudios realizados, en los que se incluyen Cámara (2009) y la tesis doctoral de Riol (2005) desarrollada en la Universidad de Ciego de Ávila.

Seguidamente se determinan las unidades de análisis, las categorías y códigos, que están relacionadas con las variables implicadas y por el sistema de categorías que previamente se ha definido, lo que permite ir agrupando y clasificando dicha información.

Se selecciona el análisis de contenido axiológico, porque describe la comunicación oral o escrita y destaca las ideas valorativas del texto. Gervilla (2000a), lo define como un conjunto de técnicas de análisis que de modo sistémico y objetivo que permite el conocimiento a profundidad de los valores presentes en cualquier universo, así como su modo de presencia.

Se utiliza con la finalidad de codificar el mensaje contenidos en la obra objeto de estudio, y hacer una valoración del escrito que nos ofrece el autor, tomando como fundamento la posibilidad de alcanzar resultados con un alto grado de objetividad y generalización por diferentes personas que se interesen por el estudio de contenido, para determinar la presencia o ausencia de valores implícitos en el documento.

\section{Muestra}

Para el análisis de contenido axiológico, son objeto de estudio cuatro cartas de las seis contenidas en el citado primer volumen. Su muestreo se realiza de forma intencional.

- Carta primera: "La impiedad es la causa del descontento individual y social”

Destaca aquí, su honda proyección humana y social subrayando la necesidad de cultivar el talento en la consecución de la verdad, lo cual queda evidenciado, cuando subraya: "el hombre nunca pierde el sentido de la justicia y el feliz impulso que lo dirige hacia la verdad" (p.13). Convencido de su actitud católica, nos dice que los hombres se desvían de la senda 
del bien y se arrojan en los brazos de la maldad a causa de estos tres males: la impiedad, la superstición y el fanatismo.

Estos tres monstruos a los que hace referencia Varela, han sido objeto de sus constantes reflexiones y han llenado su alma de amargura y, como la "amistad es el bálsamo del desconsuelo, y la comunicación de ideas el alivio de las almas sensibles" (1996, p.5), pone en su querido Elpidio los resultados de sus meditaciones.

Agrupa a los impíos en tres categorías: los que niegan la existencia de Dios; los que admiten, pero supeditada a sus caprichos e ideas; y los que aceptan tanto la existencia de Dios como sus leyes, pero se niegan a acatarlo.

Los valores sociales y afectivos son una constante en el discurso ofrecido por Varela, precisamente porque es la esencia del maravilloso ser humano que en vida fue. Plantea que la santa religión y la amable filosofía están dadas de la mano y rodeadas de paz; subraya: "(... ) la felicidad por la cual se debe luchar, (...) la esperanza de una sociedad más justa, alegre, donde la religión y la filosofía se den las manos y el conocimiento sea adquirido por todos, religiosos, ateos, pobres y ricos" (1996, p.5).

Se refiere a la paz como algo necesario para el bien de todos, como un símbolo de unidad, de libertad de expresión y de conocimiento.

- Carta tercera "Causas de la impiedad"

En esta carta, Varela le plantea a Elpidio: "investigando, querido amigo, las causas de la impiedad, creo poder reducirlas a dos clases bien distintas. Unas están en el corazón humano y otras son fruto del entendimiento" (1996, p.67).

Señala que el vicioso comienza por desatender la virtud y concluye haciéndole frente, porque la persistencia en el mal hace que llegue a considerar los remordimientos como quiméricos. Estos impíos convienen todos en oponerse a los objetos de la religión porque, dicen, no son claramente perceptibles.

Así, Varela escribe: “Adviértase solamente una conveniencia entre los dogmas, que basta para probar que no hay repugnancia entre ellos, pero nunca se puede llegar a su demostración por medios puramente naturales" (1996, p.78).

Evidentemente, la religión tiene sus dogmas que no exigen demostración, y Varela es consecuente con su pensamiento religioso, aunque va mucho más allá de esta concepción del mundo en su práctica pedagógica y sacerdotal.

Le plantea a su amigo Elpidio, en una comparación con la obra de Cervantes El Quijote, que el hombre en la esfera que esté no se despega de su locura. Compara la impiedad con la hierba seca en un jardín que, poco a poco, se va adueñando del campo sin darnos cuenta. Alerta que hay que tener cuidado de la impiedad y los grandes daños que puede causar, además de plantearnos lo que nos puede llevar hacia ella. Sella Varela su carta, con estas hermosas palabras: “¿Qué estado tan feliz el de un pueblo moral e instruido! i (...) mi más tierno afecto” (1996, p.97).

- Carta cuarta "Modo de tratar a los impíos"

Resalta aquí, un innovador sistema psicológico sobre la mejor manera de encauzar la conducta humana y, en especial, la de la juventud.

Desarrolla un método aparentemente sencillo pero extraordinariamente sabio para comprender, dirigir y encaminar a los impíos, que puede aplicarse a todos los individuos en innumerables circunstancias y a través de los tiempos, lo cual prueba su sagacidad como 
conocedor del alma, y su anticipación en esta materia en Cuba y, en el modo en que confirma estas ideas con su propia experiencia, de que los jóvenes siempre aman cuando conocen que son amados.

Asevera que la juventud propende a la justicia y que el mejor modo de tratarlos es "llevarlos con dulzura por la senda del cariño que conduce a la paz y contento" (1996, p.110). Recomienda el estudio del carácter individual de cada joven y acomodar a él nuestra conducta, y que se debe buscar que el joven sea su propio corrector.

Recomienda observar el carácter individual del joven y ajustar a él la conducta a seguir, sobre todo en la escuela. De igual forma debe hacerse con el afán de crítica que abruma a la juventud. Los jóvenes, siempre agradecen los empeños que se hacen para mejorarlos, si perciben que no se intenta oprimirlos.

Explica que ha seguido un plan que recomienda como útil y asequible. Él procede como si ya fueran lo que quieren ser, es decir, hombres ya formados; de esta manera, los jóvenes se convierten en sus colaboradores, figurándose ellos que han avanzado mucho y que ya han pasado el vértigo de las locuras juveniles. Después de formados, estos jóvenes son utilísimos, porque comprenden el valor de la estratagema.

Así trasciende el legado del maestro, sacerdote y filósofo, conformando una eticidad de raíz religiosa, válida para todos los aspectos de la vida.

- 2.1.4 Carta sexta "Furor de la impiedad"

Comenta Varela a su amado Elpidio, en esta carta, que al impío le enfurece la religión, que en vano pretende presentar como ilusión, pues es su alma misma la que dice que la ilusión es incompatible con la verdadera felicidad. Su desesperación proviene simultáneamente de su aislamiento y de su impotencia, de su engaño y humillación, como causas del furor de la impiedad.

Ahora bien, señala Varela que no debe alabarse ni a los que matan por Dios ni a los que lo hacen por ir en contra de él. Advierte también sobre otras de las causas del furor de la impiedad, y le dice a Elpidio: "No hay furor más implacable que el que proviene de la vanidad burlada. Reflexiona sobre los diversos lances de la vida humana y te convencerás sobre la exactitud de este pensamiento" (996, p.166).

Refiere Varela a la necesidad de la virtud para aplacar el furor de la impiedad. Como hombre de firme creencia, está convencido de que sobre la base de sus creencias puede asentarse la moral; simultáneamente como liberal y progresista, siente la necesidad de oponerse a los males sociales y los que se arraigan en la religión misma. Con su admonitoria elocuencia deja sentada sus ideas liberadoras, admitiendo una moral social y la libertad de conciencia; sin dejar de defender la legitimidad de la religión que tiene por única y verdadera.

\section{Procedimiento}

Con la ayuda del procesador de texto Nudist, se realiza el análisis de contenido axiológico a las cuatro epístolas escogidas. Se elige éste porque es uno de los programas más utilizados como herramienta informática para la investigación cualitativa y es adecuado para alcanzar los objetivos propuestos.

Una de las características de este programa señala Revuelta (2010), es que las categorías se pueden agrupar y organizar de forma jerárquica. Esto permite observar las relaciones que existen entre los múltiples conceptos o categorías tratadas, compararlas mediante operadores específicos para esbozar conclusiones relativas a los temas de investigación. 
Con la aplicación del procesador de texto NVivo se determinaron categorías, correspondientes en las epístolas, precisándose los códigos correspondientes a cada una de ellas. Se realiza un reporte de códigos y de texto, lo que indica que están presentes en las cartas objeto de análisis los valores políticos, morales, sociales, individuales, trascendentes, corporales, intelectuales, estéticos y temporales; con mayor énfasis, en orden, los valores: trascendentes, políticos, morales y sociales.

Para el análisis de la personalidad histórica seleccionada se siguieron algunos criterios metodológicos fundamentales:

- Relación de la obra de Félix Varela con la disciplina y materias a estudiar.

- El momento histórico en que la escribe, la fecha, el país, el género a que corresponde, las características y a que aspecto del conocimiento universal corresponde.

- Se selecciona el escrito, y se establece el vínculo de la fuente con el tema.

- Se selecciona el tipo de clases en que se debe desarrollar su estudio con tareas definidas a realizar. Ejemplo, en el seminario profundizador y el taller, para realzar la creación y aplicación del conocimiento estableciéndose el vínculo de los conocimientos de las materias y el perfil del profesional.

- En el examen final se realiza en forma de trabajo investigativo.

Se redactará un texto en forma de artículo, ensayo o ponencia, preferentemente, teniendo en cuenta:

a. Contexto histórico en que vive el autor y su incidencia en el proceso de formación de la nación y la nacionalidad cubana.

b. Contexto sociocultural en que escribe la obra y su contenido esencial.

c. Valor histórico y literario de la obra Cartas a Elpidio.

d. Los valores morales que aporta la obra

e. Trascendencia de la obra para la formación de valores en el contexto actual.

f. Importancia que le concede para su formación profesional.

Estas indicaciones hacen que el alumno profundice y active la lógica de su pensamiento realizando un análisis crítico contextualizado de la obra estudiada. Esta actividad de estudio se realiza como parte del sistema de evaluación de forma sistemática, en los temas que abordan el surgimiento y papel social de la religión, el cristianismo y las problemáticas actuales ante el crecimiento del fenómeno religioso cubano, así como el papel de las personalidades históricas en el proceso de formación de la nación y la nacionalidad cubana.

\section{Resultados}

Como se puede apreciar en los gráficos resumen, los valores que en orden jerárquico predominan son: los valores morales, los afectivos, los trascendentes y los sociales, seguido de los políticos e intelectuales, los individuales, los temporales, y los estéticos; lo cual confirma el presupuesto de que la obra Cartas a Elpidio es un verdadero tratado de ética, y que contiene un modelo educativo donde se integran como un todo orgánico un sistema de valores, susceptible de ser aprovechado en la práctica educativa. 


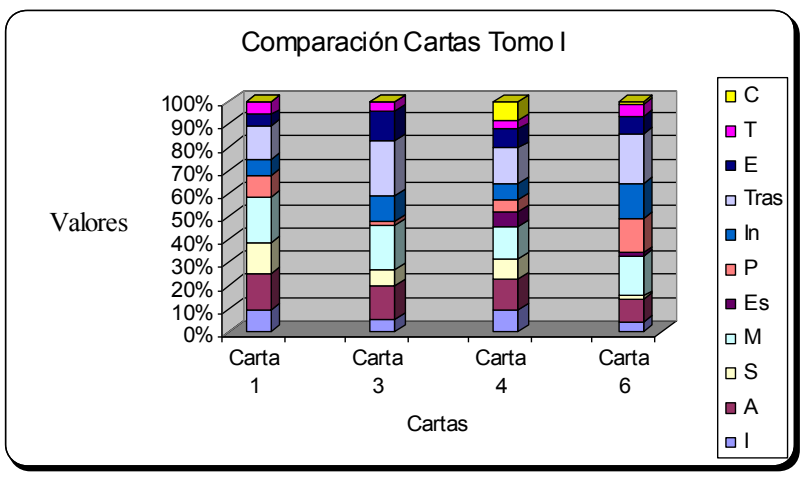

Gráfico 1. Comparación entre las cartas del Tomo I Impiedad.

El análisis porcentual realizado para indicar su presencia, así lo prueba.

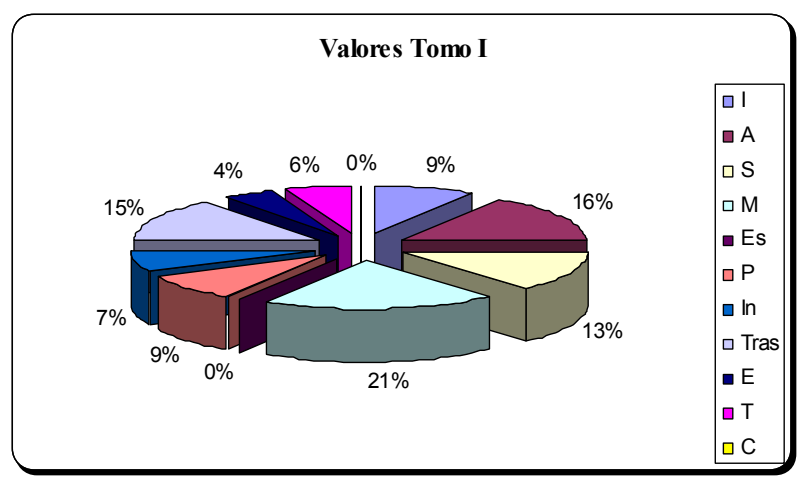

Gráfico 2. Valores contenidos en el Tomo I Impiedad.

Igualmente, se muestra en el grafico resumen los valores que alcanzan una mayor presencia, ellos son: los valores morales con el $21 \%$, los afectivos con el $16 \%$ y los trascendentes con el 15\%. Se evidencia a así una jerarquía de valores, ocupando los valores morales la supremacía.

Como se pretende formar en los educandos los valores morales a través de la instrucción, los escritos analizados ofrecen la posibilidad de ser incorporados a sus actitudes, a través del estudio de las materias filosóficas, históricas y sociopolíticas.

En el escrito sobresale la concepción del modelo pedagógico expresado en una relación de cuatro planos, de la que emergen dimensiones y cualidades.

- Educativos: enseñanza anti escolástica, científica, estimula a la reflexión, creación de un pensamiento propio.

- Psicológicos: consejos de cómo conducirse ante los jóvenes en su proceso de formación.

- Éticos-axiológico: sistema de valores (intelectuales, afectivos, sociales, morales, estéticos, políticos, individuales, trascendentales, ecológicos, temporales y corporales) que trasciende épocas.

- Culturales: distingue elementos de identidad socioculturales. 
En la obra se aprecia un modelo axiológico bien definido donde los valores morales ocupan un lugar central y coincide con las aspiraciones trazadas por el sistema educacional cubano en el proceso de formación del profesional de la educación universitaria superior. La aplicación de éste puede ser llevada a cabo en el proceso de formación.

\section{Conclusiones}

El estudio realizado corrobora la vigencia del acercamiento a la vida y obra de Félix Varela desde un análisis axiológico. La obra de Félix Varela brinda perspectivas para enfrentar los retos de la educación superior en el contexto actual, referidos esencialmente a los valores.

Mediante el análisis de contenido axiológico se puedo identificar el sistema de valores y los textos a través de los cuales se logra fundamentar la formación de valores de los educandos tomando como base el volumen primero de la obra Cartas a Elpidio, las posibilidades que ofrece su estudio en la formación de valores, lo cual pone de manifiesto la riqueza cultural y ética de sus escritos.

El sistema de clases y de evaluación precisa de acciones para implementar este modelo axiológico, en el proceso de formación del profesional.

\section{Referencias}

Alfonso, G. (2009). Diversidad, valores y vida cotidiana. [en línea], Revista Cubana de Filosofía. Edición Digital, 1, p.7. Recuperado el 2 de octubre del 2009, de http://www.filosofiacuba.org/no1/

Cámara, A. (2009). Construcción de un instrumento de categorías para analizar valores en documentos escritos. REIFOP, 12 (2), 59-72. Recuperado de http://www.aufop.com

Cámara, A. (2010). Valores en futuros profesores de Secundaria. REIFOP, 13 (3), 113-121. Recuperado de http://www.aufop.com

Cartaya, P. (1998). El legado del Padre Varela. Obra nacional de la buena prensa. México: A.C.

De Céspedes, C. M. (1999). Teología y tradiciones nacionales. Revista Enfoque, (68), 32-38.

Gallegos, E. (1997). No hay Patria sin virtud. La Habana: Editorial Unión.

Gervilla, E. (2000). Un modelo axiológico de educación integral. Revista Española de Pedagogía. Vol. 58, (215), 39-58.

Gervilla, E. (2000a). Valores de la educación integral. Bordón. Revista de Orientación Pedagógica. Vol.56, (4), pp.223-536.

Hernández, R., Fernández, C. y Batista, P. (2006). Metodología de la investigación ( $4^{\mathrm{a}}$ ed.). México: Ed. McGRAW-HILL Interamericana Editores, S. A. de C. V.

Horruitiner, P. (2006): La nueva universidad cubana: el modelo de formación. La Habana: Editorial Félix Varela.

Lolo, E. (2013). Félix Varela, José Martí, Nueva York y la Nacionalidad Cubana. Recuperado de http://www.josemarti.info/articulos/marti_varela.html

Luz y Caballero, J. de la (1981). Identificación filosófica con mi maestro. En Sánchez de Bustamante, A. Selección de textos (p.7). La Habana: Ed. Ciencias Sociales. 
Ortega, R. P. y Mínguez, V. R. (2001). Los Valores en la Educación. Recuperado de http://books.google.com.cu/books?id=

Peiró, S. (2012). Innovaciones sobre la formación inicial de profesores con relación a la educación en valores. REIFOP, 15 (1), 61-80. Recuperado de http//www.aufop.com

Pupo, R. (2008). Félix Varela en Medardo Vitier. Revista Cubana de Filosofía. Edición Digital No. 10. Septiembre - Enero 2008. Recuperado de http://revista. filosofia.cu/pensamientoc.php?id=121

Revuelta, F. I. (2010). Programas de análisis cualitativo para la investigación en espacios virtuales de formación. HYPERLINK. Recuperado de http://www.odiseame.org

Riol, M. (2005). Análisis del modelo axiológico para la formación cultural de los estudiantes universitarios a partir del pensamiento de José Martí. (Tesis doctoral inédita). Universidad de Granada, España.

Torres-Cuevas, E. (2002). Félix Varela. Los orígenes de la ciencia y conciencia cubanas. Ciudad de la Habana: Editorial Ciencias Sociales.

Varela, F. (1996). Cartas a Elpidio, sobre la impiedad, la superstición y el fanatismo en sus relaciones con la sociedad. Miami: Editorial Cubana.

Vila, E. (2005). Ética, interculturalidad y educación democrática. Hacia una pedagogía de la alteridad. Huelva, Hergué. 


\section{Autores}

María Magdalena Hernández Morales

Licenciada en Filosofía, Máster en Educación Superior. Línea investigativa: formación de valores. Departamento de Filosofía - Universidad de Ciego de Ávila, 65 200, Cuba. Teléfono: 5333 224544, magdalena@rect.unica.cu

Fuensanta Hernández Pina

Doctora en Ciencias. Departamento de Métodos de Investigación y Diagnóstico en Educación. Facultad de Educación -Universidad de Murcia, Campus de Espinardo, 30100, Murcia, España.fhpina@um.es 